\title{
Shifting Paradigms in the Medical Management of Type 2 Diabetes: Reflections on Recent Cardiovascular Outcome Trials
}

\author{
Faramarz Ismail-Beigi, $M D, P h D^{7}$, Etie Moghissi, $M D^{2}$, Mikhail Kosiborod, $M D^{3}$, and Silvio E. Inzucchi, \\ $M D^{4}$
}

'Division of Clinical and Molecular Endocrinology, Department of Medicine, Case Western Reserve University and VA Medical Center, Cleveland, $\mathrm{OH}$, USA; ${ }^{2}$ Department of Medicine, UCLA, Geffen School of Medicine, Los Angeles, CA, USA; ${ }^{3}$ Department of Cardiology, Saint Luke's Mid America Heart Institute, University of Missouri, Kansas City, MO, USA; ${ }^{4}$ Section of Endocrinology, Yale School of Medicine and Yale-New Haven Hospital, New Haven, CT, USA.

An important challenge in the management of patients with type 2 diabetes is cardiovascular disease (CVD) prevention. While it is well established that intensive glycemic control prevents the onset and slows the progression of certain microvascular complications, such a strategy utilized in multiple clinical trials over the past few decades has failed to show a similar benefit with regard to cardiovascular events, including mortality. Despite this, a major hope has been the discovery of glucose-lowering medications that simultaneously improve cardiovascular outcomes. Over the past year and a half, four randomized clinical trials (involving empagliflozin, pioglitazone, liraglutide, and semaglutide) have reported important benefits in preventing adverse cardiovascular outcomes in patients with or at risk for type 2 diabetes and established CVD. On the basis of these landmark trials, we propose that a paradigm shift in the management of patients with type 2 diabetes, specifically in those with prior macrovascular disease. A transition from current algorithms based primarily on hemoglobin Alc values to a more comprehensive strategy additionally focused on CVD prevention seems warranted.

KEY WORDS: glycemic control; individualization; MACE; hypoglycemia; heart failure; The views expressed in this article do not represent any organization or entity.

J Gen Intern Med 32(9):1044-51

DOI: $10.1007 /$ s11606-017-4061-7

(C) Society of General Internal Medicine 2017

\section{INTRODUCTION}

Among many individuals with type 2 diabetes (T2DM), an important challenge faced by both patients and the clinicians caring for them is cardiovascular disease (CVD), the main cause of excess mortality in this group. Although a strategy of intensive glycemic control aimed at reducing hemoglobin $\mathrm{A} 1 \mathrm{c}(\mathrm{HbA1c})$ to normal or near-normal levels has been shown to improve certain microvascular outcomes, to the befuddlement of many diabetes researchers and clinicians, it has failed to substantially reduce the risk of cardiovascular (CV) mortal-

Received March 3, 2017

Revised March 29, 2017

Accepted April 6, 2017

Published online May 26, 2017 ity and other adverse $\mathrm{CV}$ events such as myocardial infarction and stroke. Moreover, several glucose-lowering compounds, including some of the most widely prescribed agents, may promote adverse effects including hypoglycemia, weight gain, and fluid retention, which may have deleterious effects on the $\mathrm{CV}$ system. In addition, the traditional stepwise addition of medications over long intervals in order to achieve glycemic control may lead to prolonged periods of exposure to high glucose concentrations, increasing the risk of microvascular complications.

Ultimately, the main objectives in the management of patients with T2DM are to extend patients' survival in good health and to improve their quality of life. Therefore, what is needed in the treatment of these patients, in addition to optimal control of their glycemia, blood pressure, and lipids in a multifaceted fashion, are medications that reduce CVD events and extend life, ideally without causing high rates of hypoglycemia and/or weight gain. Indeed, the "holy grail" of T2DM therapy for several decades has been the discovery of glucoselowering medications that simultaneously improve CV outcomes. The primary focus of this article is to discuss this emerging concept in the management of T2DM, based on important data from recent $\mathrm{CV}$ outcome trials.

We propose that a paradigm shift needs to occur in the management of patients with T2DM, specifically those with established CVD. Our conventional monocular focus on the reduction of $\mathrm{HbAlc}$ should evolve into a more comprehensive approach that incorporates CVD prevention. In this new paradigm, the selection of glucose-lowering agents will be individualized based on patients' $\mathrm{CV}$ risk and their likelihood of realizing substantial clinical benefit on the basis of emerging evidence.

\section{PREVIOUS STUDIES ON THE EFFECT OF INTENSIVE GLYCEMIC CONTROL ON CVD OUTCOMES}

The most widely used glucose-lowering agent, metformin, is often thought of as being vasculoprotective, but the evidence basis is not robust. This notion stems from three comparatively small trials, including the United Kingdom Prospective Diabetes Study (UKPDS) ${ }^{1-3}$ which were conducted largely in the pre-statin era and compared metformin with diet alone or older 
sulfonylurea agents, drugs that may aggravate $\mathrm{CV}$ risk in certain settings. ${ }^{4}$ Large observational studies have also suggested potential $\mathrm{CV}$ benefits of metformin over sulfonylureas. ${ }^{5}$

Several large randomized controlled trials using intensive blood glucose control strategies aimed at the prevention of CVD in patients with newly diagnosed or well-established T2DM have failed to show any significant benefit. ${ }^{6-9}$ However, they all demonstrated prevention (and/or slowing of progression) of microvascular outcomes, especially retinopathy and nephropathy (specifically albuminuria). The UKPDS tested the effect of intensive glucose control in patients newly diagnosed with T2DM, ${ }^{6}$ while three more recent large trials enrolled older patients with established diabetes (10-12 years duration) with either prior CVD or multiple CVD risk factors. $^{7-9}$ None of these trials demonstrated any significant reduction in the risk of $\mathrm{CV}$ mortality or other adverse $\mathrm{CV}$ events, including MI, stroke, and heart failure. In fact, in the Action to Control Cardiovascular Risk in Diabetes (ACCORD) trial, there was an increase in overall mortality and in CVD-related deaths in the intensive group. ${ }^{9}$ Notably, these studies all revealed an increase in severe hypoglycemic events with more intensive glycemic control, although the relationship between these events and $\mathrm{CV}$ outcomes remains unclear. Long-term observational studies of patients enrolled in the UKPDS and the Veterans Affairs Diabetes Trial (VADT) demonstrated a modest reduction in the risk of non-fatal MI in the groups previously assigned to intensive glucose management, ${ }^{10},{ }^{11}$ while the same was not observed in extended follow-up of the ACCORD cohort. ${ }^{12}$ Importantly, none of these studies (including their long-term observational extensions) were able to reveal any significant decrease in all-cause or CV-related mortality. Although they demonstrated improvements in certain predefined microvascular outcomes (especially retinopathy), only one of these large trials showed a slight reduction in end-stage renal disease (ESRD), with a number needed to treat of 194 patients over 9.9 years to prevent one event. ${ }^{13}$

Based on this evidence, it is clear that in patients with T2DM of either short or long duration, intensive glucoselowering strategies have little or no effect on the important major challenge in T2DM management, namely prolonging survival and reducing $\mathrm{CV}$ events.

\section{RESULTS OF RECENT CV OUTCOME TRIALS USING SPECIFIC GLUCOSE-LOWERING MEDICATIONS}

In 2008, the US Food and Drug Administration (FDA) published a Guidance for Industry focused on the CV safety of glycemia-lowering drugs, and as a result, the past decade has seen a notable increase in the number and size of $\mathrm{CV}$ outcome trials evaluating glucose-lowering compounds for treatment of T2DM. The primary focus of these studies has been CV safety, leading to the selection of patients at high risk for CVD and intentional minimization of differences in glycemia between drug and placebo. Thus an agent would have to exhibit a marked pleiotropic (and presumably non-glycemic) effect in order to yield a significant CVD benefit within the short time course of a typical CV safety trial. It is not surprising, then, that a number of these studies ${ }^{14-17}$ demonstrated what they were primarily designed to demonstrate-non-inferiority compared to placebo and confirmation of $\mathrm{CV}$ safety-but showed no clinical benefit beyond HbAlc lowering. The hope that some of the agents would produce meaningful clinical CV benefits in addition to glycemic control remained unfulfilled.

In the last year and a half, however, we have witnessed four major clinical trials that have found a convincing benefit of several glucose-lowering medications on $\mathrm{CV}$ outcomes, each without increasing the risk of hypoglycemia. Importantly, in two of these studies, there were significant reductions in $\mathrm{CV}$ and total mortality.

We will briefly review the results of these studies, including recent data on the use of a peroxisome proliferator-activated receptor (PPAR) gamma agonist (pioglitazone), a sodium glucose cotransporter-2 (SGLT2) inhibitor (empagliflozin), and two glucagon-like peptide-1 (GLP-1) receptor agonists (liraglutide and semaglutide). These data address a major challenge in the care of the patient with T2DM-improving CV outcomes. Important baseline characteristics of patients enrolled in these studies, along with the primary and all-cause mortality and CVD-related secondary outcomes, are summarized in Table 1.

\section{Pioglitazone}

Thiazolidinediones (TZDs) exhibit potent insulin-sensitizing properties and reduce $\mathrm{HbAlc}$ without increasing hypoglycemia. Insulin resistance, a very common finding in T2DM, was for decades associated with increased $\mathrm{CV}$ risk irrespective of the presence or absence of hyperglycemia. ${ }^{23}$ The Prospective Pioglitazone Clinical Trial in Macrovascular Events (PROactive) in 2005 found that pioglitazone (a TZD) reduced the secondary outcome of major CV adverse events (a composite of all-cause mortality, non-fatal MI, and non-fatal stroke) in patients with T2DM with established CVD. ${ }^{24}$ However, its primary outcome, a broader composite outcome that included peripheral vascular disease events, was not significantly reduced. Also, the use of pioglitazone resulted in a higher rate of heart failure hospitalizations, calling into question the overall $\mathrm{CV}$ risk-benefit ratio of this therapy. Subsequently, data emerged suggesting that another TZD, rosiglitazone, might increase the risk of myocardial infarction $^{25}$ (a suggestion that was not confirmed by a follow-up large randomized trial ${ }^{26}$ ), that both TZDs increased fracture rates in women, and that pioglitazone might be associated with an increased risk for bladder cancer. As a result, the once popular TZDs were relegated to almost niche status.

In the midst of this uncertainty, the Insulin Resistance Intervention after Stroke (IRIS) trial was under way. ${ }^{19}$ Previous data had linked increased risk for stroke with insulin resistance even in individuals without diabetes. ${ }^{27}$ Post hoc analysis of the PROactive trial showed that use of pioglitazone resulted in a 
Table 1 Baseline Characteristics of Participants, Primary Outcome, All-Cause Mortality, and CVD-Related Secondary Outcomes

\begin{tabular}{|c|c|c|c|c|c|c|c|c|}
\hline & \multicolumn{2}{|c|}{$\begin{array}{l}\text { EMPA-REG OUTCOME } \\
N=7020\end{array}$} & \multicolumn{2}{|l|}{$\begin{array}{l}\text { IRIS } \\
N=3876\end{array}$} & \multicolumn{2}{|l|}{$\begin{array}{l}\text { LEADER } \\
N=9340\end{array}$} & \multicolumn{2}{|l|}{$\begin{array}{l}\text { SUSTAIN-6 } \\
N=3297\end{array}$} \\
\hline & $\begin{array}{l}\text { Empagliflozin } \\
N=4687\end{array}$ & $\begin{array}{l}\text { Placebo } \\
N=\mathbf{2 3 3 3}\end{array}$ & $\begin{array}{l}\text { Pioglitazone } \\
N=1939\end{array}$ & $\begin{array}{l}\text { Placebo } \\
N=1937\end{array}$ & $\begin{array}{l}\text { Liraglutide } \\
N=4668\end{array}$ & $\begin{array}{l}\text { Placebo } \\
N=4672\end{array}$ & $\begin{array}{l}\text { Semaglutide } \\
N=1648\end{array}$ & $\begin{array}{l}\text { Placebo } \\
N=1649\end{array}$ \\
\hline \multicolumn{9}{|l|}{ Baseline features } \\
\hline Age (years) & $63.1 \pm 8.6$ & $63.2 \pm 8.8$ & $63.5 \pm 10.6$ & $63.5 \pm 10.7$ & $64.2 \pm 7.2$ & $64.4 \pm 7.2$ & $64.7 \pm 7.2 * *$ & $64.6 \pm 7.6 * *$ \\
\hline Male sex (\%) & 71.2 & 72.0 & 66.7 & 64.3 & 64.5 & 64.0 & 61.5 & 60.0 \\
\hline White race $(\%)$ & 72.6 & 71.9 & $83.9 *$ & $85.0 *$ & $77.5^{*}$ & $77.5^{+}$ & 84.0 & 82.0 \\
\hline BMI $\left(\mathrm{kg} / \mathrm{m}^{2}\right)$ & $30.6 \pm 5.3$ & $30.7 \pm 5.2$ & $29.9 \pm 5.6$ & $30.0 \pm 5.3$ & $32.5 \pm 6.3$ & $32.5 \pm 6.3$ & $32.8 \pm 6.2 * *$ & $32.8 \pm 6.2 * *$ \\
\hline $\operatorname{HbA} 1 \mathrm{c}(\%)$ & $8.1 \pm 0.9$ & $8.1 \pm 0.8$ & $5.8 \pm 0.4$ & $5.8 \pm 0.4$ & $8.7 \pm 1.6$ & $8.2 \pm 1.5$ & $8.1 \pm 1.5^{* *}$ & $8.7 \pm 1.5^{* *}$ \\
\hline Prevalent CVD (\%) & 99.4 & 98.9 & $99.9 *$ & $99.8 *$ & 82.1 & 80.6 & 82.1 & 83.8 \\
\hline Heart failure (\%) & 9.9 & 10.5 & $0.8^{*}$ & $0.5^{*}$ & 17.9 & 17.8 & 23.1 & 24.0 \\
\hline Metformin therapy (\%) & 73.8 & 74.3 & $0.1 *$ & $0.2^{*}$ & 75.8 & 77.1 & 73.5 & 73.0 \\
\hline Insulin therapy $(\%)$ & 48.0 & 48.6 & $0.0^{*}$ & $0.0^{*}$ & 43.7 & 45.6 & 58.0 & 58.0 \\
\hline $\begin{array}{l}\text { Blood pressure } \\
(\mathrm{mmHg})\end{array}$ & $\begin{array}{l}135 / 77 \\
( \pm 17 / 10)\end{array}$ & $\begin{array}{l}136 / 77 \\
( \pm 17 / 10)\end{array}$ & $\begin{array}{l}133 / 79 \\
( \pm 18 / 11)\end{array}$ & $\begin{array}{l}133 / 79 \\
( \pm 17 / 11)\end{array}$ & $\begin{array}{l}136 / 77 \\
( \pm 18 / 10)\end{array}$ & $\begin{array}{l}136 / 77 \\
( \pm 18 / 10)\end{array}$ & $\begin{array}{l}136 / 77 \\
( \pm 17 / 10) * *\end{array}$ & $\begin{array}{l}135 / 77 \\
( \pm 17 / 10) * *\end{array}$ \\
\hline ACEI or ARB (\%) & 81.0 & 80.1 & 56.4 & 54.6 & $83.7^{\S}$ & $82.1^{\S}$ & $83.6^{\S}$ & $83.4^{\S}$ \\
\hline $\begin{array}{l}\text { LDL-cholesterol } \\
\text { (mg/dl) }\end{array}$ & $85.9 \pm 36$ & $\begin{array}{l}84.9 \pm \\
35.3\end{array}$ & $87.6 \pm 31.5$ & $87.9 \pm 31.5$ & $\begin{array}{l}90.0^{ \pm} \\
36.5^{ \pm}\end{array}$ & $\begin{array}{l}90.1 \pm \\
36.1^{\ddagger}\end{array}$ & $\begin{array}{l}82.5 \pm \\
44.2 * *\end{array}$ & $\begin{array}{l}82.3 \pm \\
47.0^{* *}\end{array}$ \\
\hline Statin therapy $(\%)$ & 77.4 & 76.0 & 82.5 & 82.4 & 72.9 & 71.4 & 72.8 & 72.3 \\
\hline Aspirin therapy (\%) & 82.7 & 82.6 & 92.0 & 92.3 & 63.8 & 62.1 & 63.8 & 64.3 \\
\hline eGFR <60 (\%) & 26.0 & 25.9 & NR & NR & 23.9 & 22.3 & 28.5 & 28.5 \\
\hline Albuminuria (\%) & 39.5 & 39.8 & NR & NR & $36.4^{\|}$ & $37.6^{\|}$ & NR & NR \\
\hline \multicolumn{9}{|l|}{ Main trial outcomes } \\
\hline Primary outcome & \multicolumn{2}{|c|}{$\begin{array}{l}\text { 3-Point MACE } \\
\mathrm{HR}=0.86(0.74-0.99)\end{array}$} & \multicolumn{2}{|c|}{$\begin{array}{l}\text { Fatal/non-fatal MI or stroke } \\
\mathrm{HR}=0.76(0.62-0.93)\end{array}$} & \multicolumn{2}{|c|}{$\begin{array}{l}\text { 3-Point MACE } \\
\text { HR = 0.87 } \\
(95 \% \text { CI, } 0.78-0.97)\end{array}$} & \multicolumn{2}{|c|}{$\begin{array}{l}\text { 3-Point MACE } \\
\text { HR = 0.74 } \\
(95 \% \text { CI, 0.58-0.95) }\end{array}$} \\
\hline \multicolumn{9}{|l|}{ Secondary outcomes } \\
\hline CV mortality & \multicolumn{2}{|l|}{$0.62(0.49-0.77)$} & \multicolumn{2}{|c|}{$0.79(0.48,1.31)^{\dagger}$} & \multicolumn{2}{|c|}{$0.78(0.66-0.93)$} & \multicolumn{2}{|c|}{$0.98(0.65-1.48)$} \\
\hline All-cause mortality & \multirow{2}{*}{\multicolumn{2}{|c|}{$\begin{array}{l}0.68(0.57-0.82) \\
0.87(0.70-1.09)\end{array}$}} & \multicolumn{2}{|c|}{$0.93(0.73-1.17)$} & \multicolumn{2}{|c|}{$0.85(0.74-0.97)$} & \multicolumn{2}{|c|}{$1.05(0.74-1.50)_{\pi}$} \\
\hline MI & & & $0.87(0.70-1$. & & & & $0.74(0.51-1.0$ & \\
\hline Stroke & \multicolumn{2}{|l|}{$1.18(0.89-1.56)$} & \multicolumn{2}{|c|}{$0.82(0.61-1.10)$} & \multicolumn{2}{|c|}{$0.89(0.72-1.11)$} & \multicolumn{2}{|c|}{$0.61(0.38-0.99)^{\mathbb{I I}}$} \\
\hline HF hospitalization & \multicolumn{2}{|l|}{$0.65(0.50-0.85)$} & \multicolumn{2}{|c|}{$1.22(0.81-1.84)^{\dagger}$} & \multicolumn{2}{|c|}{$0.87(0.73-1.05)$} & \multicolumn{2}{|c|}{$1.11(0.77-1.61)$} \\
\hline
\end{tabular}

Data are from the following trials: EMPA-REG OUTCOME, ${ }^{18}$ IRIS,${ }^{19}$ LEADER, ${ }^{20}$ and SUSTAIN-6. ${ }^{21}$

$N=$ number $C V D=$ cardiovascular disease; $A C E I=$ angiotensin-converting enzyme inhibitor; $A R B=$ angiotensin receptor blocker; $L D L=$ lowdensity lipoprotein; $e G F R=$ estimated glomerular filtration rate; $M A C E=$ major adverse cardiovascular events; $H F=$ heart failure; $N R=n o t$ reported *Personal communication, C. Viscoli, January 23, 2017

II IRIS, the endpoint definitions varied slightly from those of the other trials. The data shown are for the pre-specified adjudicated outcomes closest to CV mortality ("fatal MI or stroke"), MI ("acute coronary syndrome [ACS]"), and HF hospitalization ("serious HF")

${ }^{*}$ Novo Nordisk LEADER clinical trial report

${ }^{S}$ Percentages do not account for dual ACEI-ARB therapy

"Mann J. Kidney Week 2016, November 15-20, 2016, Chicago, IL ${ }^{22}$

"Non-fatal events only

**Values approximated from individual dosing groups presented in original manuscript

major reduction in new stroke events in participants with prior stroke at enrollment, ${ }^{28}$ and reduced the rates of fatal and nonfatal MI in those with prior MI at trial entry. ${ }^{29}$

In the IRIS trial, 3876 non-diabetic patients with recent stroke or transient ischemic attack (TIA) and insulin resistance based on the homeostasis model equation (HOMA > 3.0) were randomized to pioglitazone (titrated up to $45 \mathrm{mg}$ daily) or placebo. The mean follow-up was nearly 5 years. ${ }^{19}$ Participants were older (mean age 63.5 years), and were mostly overweight or obese (mean BMI of approximately $30 \mathrm{~kg} / \mathrm{m}^{2}$ ). Mean fasting plasma glucose was $98 \mathrm{mg} / \mathrm{dl}$ and mean $\mathrm{HbAlc}$ was $5.8 \%$; a majority of the study cohort had prediabetes.

The primary outcome was time to first event for fatal and non-fatal stroke or myocardial infarction (MI), which was reduced in patients on active therapy from $11.8 \%$ to $9.0 \%$ $(\mathrm{HR}=0.24 ; p=0.007)$. A key secondary endpoint of progression to $\mathrm{T} 2 \mathrm{DM}$ was reduced from $7.7 \%$ to $3.8 \%(\mathrm{HR}=0.48$; $p<0.001),{ }^{30}$ confirming a potent diabetes prevention effect of the class, as had been shown by others. ${ }^{31}$

As in prior studies, pioglitazone was associated with certain side effects, including weight gain and edema. Serious bone fractures were also more common in those randomized to pioglitazone ( $5.1 \%$ vs. $3.2 \%, p=0.003$ ). There was no increase in the risk for any cancer. Heart failure was not significantly increased, perhaps due to the exclusion of patients with established heart failure at enrollment and a dose adjustment algorithm for patients who developed weight gain or edema.

These data appeared to confirm the results of PROactive from more than 10 years earlier showing that this TZD had a positive effect on atherosclerotic CV outcomes in patients with established CVD, with IRIS extending this observation to insulin-resistant but still non-diabetic (and often prediabetic) patients. 


\section{Empagliflozin}

SGLT2 inhibitors are relatively new glucose-lowering agents that promote urinary glucose excretion ${ }^{32}$; they rarely cause hypoglycemia unless used in combination with sulfonylureas or insulin. In addition, these drugs have modest beneficial effects on systolic blood pressure, likely related to their diuretic properties, and on body weight, resulting from chronic calorie loss. In the first $\mathrm{CV}$ outcome trial to report with this category of medications, EMPA-REG OUTCOME, ${ }^{18}$ empagliflozin showed a modest reduction in the primary composite outcome of death from CV causes, nonfatal MI, or nonfatal stroke (major adverse cardiovascular events; MACE) from $12.5 \%$ to $10.5 \%$ (HR $=0.86[p=0.04])$. Although not an extraordinary degree of risk reduction, this was still notable, because it represented the first time that any single diabetes drug had unequivocally improved overall CV outcomes in a large trial involving high-risk individuals. Even more impressive was the fact that the component that drove the overall reduction in MACE was CV mortality, which was markedly reduced, from $5.9 \%$ to $3.7 \%$ ( $\mathrm{HR}=0.62[p<0.0001]$ ). Allcause mortality was also cut by $32 \%(p<0.0001)$ with active therapy. The other MACE components (non-fatal MI $[\mathrm{HR}=0.87 ; p=0.22]$ and non-fatal stroke $[\mathrm{HR}=1.24$; $p=0.16])$ were not significantly affected. Among several secondary endpoints, notable was a significant $35 \%$ reduction in heart failure hospitalization. In a subsequent report, it appeared that the reduced incidence of hospitalization due to heart failure was seen in patients both with and without established heart failure at baseline, suggesting that the drug may not only improve the progression of heart failure but could also prevent its development. ${ }^{33}$ All the CV benefits occurred, somewhat surprisingly, within a short time period, with event curve divergence within 1-2 months, and persisted over the median follow-up of 3.1 years. More recently reported was another clinically significant secondary outcome: a reduction in incident or worsening nephropathy (composite of progression to macroalbuminuria, doubling of serum creatinine level, initiation of renal replacement therapy, death from renal disease), from $18.8 \%$ to $12.7 \%(\mathrm{HR}=0.61$; $p<0.0010){ }^{34}$

Based on these findings, the US FDA recently allowed a label change for this compound, now including a new indication for the reduction in $\mathrm{CV}$ mortality in patients with $\mathrm{T} 2 \mathrm{DM}$ and established CVD. The American Diabetes Association (ADA) also recently recommended consideration of its use in this subgroup of patients. ${ }^{35}$ Additional CV outcome trials with two other SGLT2 inhibitors, canagliflozin and dapagliflozin, are currently under way and include patients both with and without prior CVD.

There is much speculation concerning the possible mechanisms underlying these benefits, but few data exist. The early separation of the event curves for CV mortality and heart failure hospitalization suggests that the effect is not mediated through slowing of the atherosclerotic process. It is tempting to consider that the $\mathrm{CV}$ mortality benefits were simply due to the diuretic properties of this SGLT2 inhibitor, particularly in light of the positive heart failure outcomes. However, the vast majority of the trial participants did not have heart failure at baseline, and death directly related to heart failure constituted less than $10 \%$ of all fatal CV outcomes. What is clear, however, is that the observed $\mathrm{CV}$ and renal benefits of empagliflozin had little to do with glucose lowering, given the minor difference in $\mathrm{HbAlc}$ between the empagliflozinand placebo-treated patients during the study (approximately $-0.4 \%)$.

\section{Liraglutide and Semaglutide}

GLP-1 receptor agonists are injectable agents that lower blood glucose via several mechanisms: they increase glucosedependent insulin secretion, lower postprandial glucagon levels, slow gastric emptying, and cause satiety and reduced food intake. ${ }^{36}$ They do not, however, increase the risk of hypoglycemia unless used in combination with sulfonylureas or insulin. Additional potential benefits include weight loss and a modest reduction in systolic blood pressure.

The Liraglutide Effect and Action in Diabetes: Evaluation of Cardiovascular Outcome Results (LEADER) trial was a multi-center placebo-controlled trial that evaluated the effects of liraglutide, a once daily GLP-1 receptor agonist, on CV outcomes in patients with T2DM. ${ }^{20}$ Two key subgroups of patients were included: those aged 50 years or older with established CVD, and those aged 60 years or older with at least one risk factor for CVD. Patients were randomly assigned to $1.8 \mathrm{mg}$ liraglutide or matching placebo administered subcutaneously, with a median follow-up of 3.8 years. The primary endpoint was time to the first MACE event.

Liraglutide demonstrated a modest reduction of the primary MACE endpoint, from $14.9 \%$ to $13.0 \%$ (HR $=0.87 ; p=0.01$ ). Importantly, the rates of $\mathrm{CV}$ deaths and all-cause mortality were also reduced in liraglutide-treated patients, from $6.0 \%$ to $4.7 \%$ $(\mathrm{HR}=0.88 ; p=0.007)$ and from $9.6 \%$ to $8.2 \%(\mathrm{HR}=0.85$; $p=0.02$ ), respectively. Rates of non-fatal MI and non-fatal stroke and hospitalizations for heart failure were not significantly affected, however. Use of the agent was also associated with decreased risk of new onset macroalbuminuria. Of note, the ADA also recently recommended consideration of the use of this GLP-1 receptor agonist in patients with $\mathrm{T} 2 \mathrm{DM}$ and history of $\mathrm{CVD}{ }^{35}$

The Trial to Evaluate Cardiovascular and Other Long-term Outcomes with Semaglutide in Subjects with Type 2 Diabetes (SUSTAIN-6) was a multi-center, randomized, placebocontrolled trial designed to evaluate the effects of semaglutide - an investigational once-weekly GLP-1 receptor agonist - on CV outcomes in patients with T2DM. ${ }^{21}$ The primary outcome here was also time to the first MACE event. Patients were stratified into two key subgroups similar to the LEADER trial (see above), and were randomly assigned to either $0.5 \mathrm{mg}$ or $1.0 \mathrm{mg}$ once-weekly semaglutide or placebo, administered subcutaneously, and were followed for a median of 2.1 years.

Semaglutide significantly reduced the primary MACE outcome, from $8.9 \%$ to $6.6 \%$ (HR $=0.74 ; p=0.02)$, due to an 
additive beneficial effect on non-fatal MI $(\mathrm{HR}=0.84$; $p=0.12)$ and non-fatal stroke ( $\mathrm{HR}=0.61 ; p=0.04)$. However, there was no significant difference between semaglutide and placebo in rates of $\mathrm{CV}$ death or all-cause mortality. Use of the agent was also associated with decreased risk of new onset macroalbuminuria. However, for unclear reason, rates of retinopathy complications were significantly higher with semaglutide than with placebo $(\mathrm{HR}=1.76 ; p=0.02)$.

Similar to the results of the EMPA-REG OUTCOME trial, the mechanism(s) of $\mathrm{CV}$ benefit in the LEADER and SUSTAIN-6 trials are not known; however, a few observations and comparisons are worth pointing out. The reductions in $\mathrm{CV}$ and all-cause mortality using liraglutide were less profound than those observed with empagliflozin, and took considerably longer to emerge. These findings, along with the fact that all components of the MACE composite and the secondary endpoint of coronary revascularization trended in favor of liraglutide, suggest that, in contrast to empagliflozin, the mechanisms of CV benefit from the GLP-1 receptor agonists involve atherosclerosis. The same may be said for semaglutide in SUSTAIN-6, with effects on MACE emerging after 12 months of follow-up. In addition, although caution must be exercised when interpreting subgroup analyses, the benefit of both liraglutide and semaglutide on the primary endpoint was significant only in the pre-specified subgroup of patients with established CVD, and not in those with risk factors only.

\section{Summary of Effects of the Above Agents}

Figure 1 lists the major effects of the medications used in the above trials. Importantly, the four medications reviewed all reduce CVD events in persons with a history of CVD. Two compounds (empagliflozin and liraglutide) significantly reduced $\mathrm{CV}$ and all-cause mortality. Hospitalization due to heart failure was increased with the use of pioglitazone in earlier trials, but this was not observed in IRIS, which, as mentioned, excluded those with heart failure at enrollment and allowed for dose down-titration for fluid retention-related side effects. Hospitalization for heart failure was less frequently with empagliflozin, and there was a neutral effect with liraglutide and semaglutide. All four agents are associated with fewer hypoglycemic events compared to prior experience with sulfonylureas and insulin. With the exception of pioglitazone, the medications discussed also reduce body weight but are costly.

\section{CONCLUSIONS}

Treatment of T2DM is undergoing a major transformation that holds the promise of improved outcomes for patients. For decades, more intensive glucose control strategies have failed to positively impact what are important outcomes for this group, namely $\mathrm{CV}$ events and mortality. Now, in less than a 2 year span of time, four large clinical trials evaluating four different glucose-lowering drugs have convincingly demonstrated CV benefits. Moreover, taken in the context of previous CVD outcome studies in patients with T2DM undergoing intensification of glucose control, achieving relatively large $\mathrm{HbAlc}$ reductions up to $1.6 \%$, the effect of these medications is almost certainly mediated by mechanisms other than glucose lowering, given the comparatively modest decreases in $\mathrm{HbA1c}$ observed in these studies between the active therapy and placebo groups.

Based on the above recent findings, the time may have arrived for a paradigm shift in our thinking and our approach to the management of T2DM, with a focus not only on effective glycemic control, but also on whether the agents reduce CV risk. Figure 2 shows the spectrum of CVD as manifested by patients with T2DM and the corresponding clinical trial evidence involving the seven major categories of glucose-lowering drugs. Because the main goal in treating patients with T2DM is to prolong healthy life and improve its quality, and since CVD is the leading cause of increased mortality and morbidity in patients with T2DM, glycemialowering treatments that have been proven to lower CV and all-cause mortality and to reduce CV complications should now be prioritized in individuals with overt CVD.

Therefore, the current algorithms for management of $\mathrm{T} 2 \mathrm{DM}$ that are based primarily on $\mathrm{HbA} 1 \mathrm{c}$ values ought to shift towards a new paradigm - one in which the patients' cardiovascular risk and their likelihood of realizing a CVD benefit are incorporated into the glucose-lowering drug

\begin{tabular}{|c|c|c|c|c|c|c|c|}
\hline Agent & MACE* $^{\star}$ & $\begin{array}{c}\text { CVD } \\
\text { mortality }\end{array}$ & $\begin{array}{l}\text { All-cause } \\
\text { mortality }\end{array}$ & $\begin{array}{c}\mathrm{HF} \\
\text { admissions } \dagger\end{array}$ & $\begin{array}{c}\text { Hypoglycemia } \\
\text { risk }\end{array}$ & $\begin{array}{l}\text { Weight } \\
\text { change }\end{array}$ & Cost \\
\hline Pioglitazone & $\sqrt{ }$ & $\Leftrightarrow$ & $\Leftrightarrow$ & 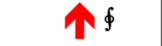 & Low & & Low \\
\hline Empagliflozin & & 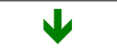 & 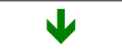 & $v$ & Low & & High \\
\hline Liraglutide & $\mathrm{l}$ & 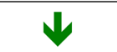 & b & $\Leftrightarrow$ & Low & & High \\
\hline Semaglutide & $\sqrt{k}$ & $\Leftrightarrow$ & $\Leftrightarrow$ & $\Leftrightarrow$ & Low & $\sqrt{6}$ & High $^{a}$ \\
\hline
\end{tabular}

Figure 1 Risk of CVD outcomes, CVD-related and all-cause mortality, key side effects, and cost associated with use of listed agents. Data are from the following trials: IRIS (pioglitazone), ${ }^{19}$ EMPA-REG OUTCOME (empagliflozin), ${ }^{18}$ LEADER (liraglutide), ${ }^{20}$ and SUSTAIN-6 (semaglutide). ${ }^{21}$ Downward arrows (green) indicate a reduction, and upward arrows (red) indicate an increase; horizontal arrows (yellow) indicate neutral effect. *Denotes major adverse cardiovascular events, most commonly a composite of cardiovascular death, nonfatal MI, and nonfatal stroke. ${ }^{\dagger}$ Denotes hospitalization due to heart failure. ${ }^{*}$ Risk for severe hypoglycemia is compared to that observed in patients using sulfonylureas or insulin. ${ }^{\oint}$ Based on several studies using pioglitazone (excluding IRIS). ${ }^{\text {a }}$ Cost assumed since drug is not yet marketed. 


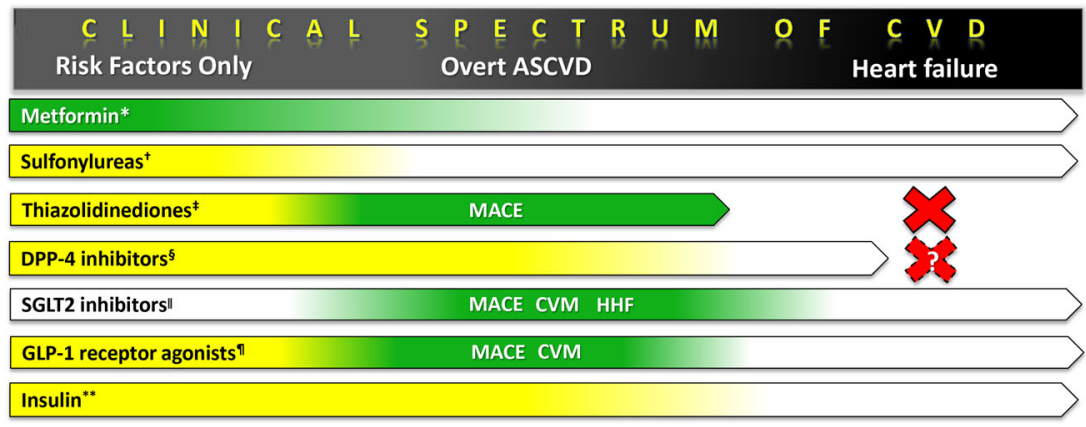

Figure 2 Indications and CV evidence of glucose-lowering agents in type 2 diabetes. Arrow bar denotes patient category in which the medication class is currently indicated. Green indicates effectiveness (i.e., reduced CV events), yellow indicates CV neutrality, and no color indicates lack of $\mathrm{CV}$ data from randomized clinical trials, as interpreted by the authors. For $\mathrm{CV}$ effectiveness, the specific types of events reduced are also listed (MACE = major adverse CV events; $\mathrm{CVM}=\mathrm{CV}$ mortality; $\mathrm{HHF}=$ hospitalization for heart failure.) $*$ Metformin effectiveness demonstrated in UKPDS-34 $(n=1704),{ }^{1}$ Kooy et al. $(n=390),{ }^{2}$ and SPREAD-DIMCAD $(n=304) .{ }^{3}$ Sulfonylurea safety demonstrated for glibenclamide and chlorpropamide in UKPDS-33 $(n=3867) .{ }^{*}$ For thiazolidinediones, safety shown for rosiglitazone for patients with CV risk factors $(\mathrm{RECORD,}$ $n=4447)^{25}$ and effectiveness shown for pioglitazone in PROactive $(n=5238)^{23}$ and IRIS (insulin-resistant stroke population with no diabetes, $n=3876$.) ${ }^{19}$ Contraindicated in heart failure. ${ }^{\S}$ Dipeptidyl peptidase-4 (DPP-4) inhibitor safety shown for saxagliptin (SAVOR-TIMI 53, $n=16,492),{ }^{14}$ alogliptin (EXAMINE, $\left.n=5380\right),{ }^{15}$ and sitagliptin (TECOS, $\left.n=14,671\right) .{ }^{16}$ SAVOR found an increased HHF with saxagliptin, with a similar trend in EXAMINE; current guidelines caution the use of saxagliptin and alogliptin in heart failure patients. " SGLT2 inhibitor effectiveness demonstrated for empagliflozin in EMPA-REG OUTCOME $(n=7020)^{18}$; although HHF was reduced in that study, the drug has not yet been tested in a dedicated heart failure study. ${ }^{\top}$ Only GLP-1 receptor agonist effectiveness demonstrated for liraglutide (MACE, CVM) in LEADER $(n=9340)^{20}$ and the investigational semaglutide (MACE only) in SUSTAIN-6 $(n=3297) .^{21 *}$ Insulin safety shown in UKPDS-33 $(n=3867)^{6}$ and ORIGIN $(n=12,537) .{ }^{37}$ Acute in-hospital studies are not considered.

selection process. Guideline committees will need to consider whether agents proven to improve important clinical outcomes, including mortality, should now be preferred as a secondary prevention strategy in patients with T2DM and established CVD. An opinion piece with views similar to those expressed in this article was published recently. ${ }^{38}$

We look forward to the results of studies with these and other SGLT2 inhibitors and GLP-1 receptor agonists to better understand whether the improved outcomes found thus far might also apply to other members of these medication classes. In addition, we anticipate results of the next generation of trials using these drugs at earlier stages of T2DM, especially in patients without prevalent CVD. Further study is also now needed to explore the effects on hard clinical outcomes of combining classes of compounds with possible complementary benefits. ${ }^{23}$ Of course, a rate-limiting step in the implementation of any new recommendations favoring non-generic medications is their high cost. We encourage the pharmaceutical industry and pharmaceutical benefit plans to work with insurers to assist large populations of patients with T2DM and CVD in improving their access to these treamtents.

As always, individualization of goals and therapy should continue to play a central role in decision-making. In choosing a therapeutic regimen, we should continue to consider, in addition to prevalent CVD, each patient's capabilities, finances, living situation, support systems, cognitive status, other comorbidities, and life expectancy, while implementing shared decision-making. ${ }^{39},{ }^{40}$ However, we expect that applying an updated paradigm for the management of T2DM in clinical practice will likely result in longer and healthier survival for patients afflicted with this disease.
Corresponding Author: Faramarz Ismail-Beigi, $M D, P h D$; Division of Clinical and Molecular Endocrinology, Department of MedicineCase Western Reserve University and VA Medical Center, 10900 Euclid Ave., Cleveland, $\mathrm{OH}$ 44106-4951, USA (e-mail: fxi2@case.edu).

Author Contributions: F. Ismail-Beigi wrote the first draft. All authors played an active role in data collection and in multiple revisions of the manuscript; all played an active role in creating Table 1 and Figs. 1 and 2. All authors have reviewed the manuscript and approve its entire contents.

\section{Compliance with Ethical Standards:}

Conflict of Interest: Dr. Ismail-Beigi has received grants from NIH and Novo Nordisk to conduct clinical trials. He has shares in Thermalin Diabetes, Inc., and serves as a consultant for Sanofi and COVANCE. Dr. Moghissi has served as a speaker/consultant for AstraZeneca, Boehringer Ingelheim, Eli Lilly, Jansen, Merck, and Novo Nordisk.

Dr. Kosiborod has research grants from AstraZeneca and Boehringer Ingelheim. He serves as a consultant/advisory board member for AstraZeneca, Amgen, Eli Lilly, Boehringer Ingelheim, GSK, Sanofi, Novo Nordisk, Merck (Diabetes), ZS Pharma, and Glytec, and is on the speakers bureau of Amgen.

Dr. Inzucchi has served as a consultant to Merck, Janssen, Sanofi/ Lexicon, and vTv Therapeutics. He has participated on clinical trial steering or publications committees for Boehringer Ingelheim, AstraZeneca, and Daiichi Sankyo. He has served as a member of data monitoring committees for Novo Nordisk and Intarcia.

All remaining authors declare that they do not have a conflict of interest.

\section{ITEMS FOR THE BOX}

1. A major challenge faced by patients with type 2 diabetes and clinicians caring for them is cardiovascular disease (CVD), the main cause of excess mortality in this group.

2. More intensive glycemic control improves certain microvascular outcomes but has not substantially reduced the 
risk of cardiovascular (CV) mortality and other adverse $\mathrm{CV}$ events such as myocardial infarction and stroke.

3. Based on the results of recent trials, the use of medications now proven to reduce $\mathrm{CV}$ complications should be prioritized in patients with established CVD, while continuing a multifaceted approach for controlling hypertension and dyslipidemia.

4. We anticipate future trials using SGLT2 inhibitors or GLP-1 receptor agonists at earlier stages of type 2 diabetes, especially in those without prevalent CVD.

5. Current algorithms for the management of type 2 diabetes based primarily on $\mathrm{HbAlc}$ values ought to shift towards a new paradigm that incorporates patients' $\mathrm{CV}$ risk and their likelihood of realizing a CVD benefit into the glucose-lowering drug selection process.

\section{REFERENCES}

1. UK Prospective Diabetes Study (UKPDS) Group. Effect of intensive bloodglucose control with metformin on complications in overweight patients with type 2 diabetes (UKPDS 34). Lancet 1998;352:854-65.

2. Kooy A, de Jager J, Lehert P, et al. Long-term effects of metformin on metabolism and microvascular and macrovascular disease in patients with type 2 diabetes mellitus. Arch Intern Med 2009;169:616-25.

3. Hong J, Zhang Y, Lai S, et al. SPREAD-DIMCAD Investigators. Effects of metformin versus glipizide on cardiovascular outcomes in patients with type 2 diabetes and coronary artery disease. Diabetes Care 2013;36:1304-11

4. Abdelmoneim AS, Eurich DT, Light PE, et al. Cardiovascular safety of sulphonylureas: over 40 years of continuous controversy without an answer. Diabetes Obes Metab 2015;17(6):523-32.

5. Maruthur NM, Tseng E, Huftless S, et al. Diabetes medications as monotherapy or metformin-based combination therapy for type 2 diabetes: a systemic review and meta-analysis. Ann Intern Med 2016;164:740-51

6. UK Prospective Diabetes Study (UKPDS) Group. Intensive blood-glucose control with sulphonylureas or insulin compared with conventional treatment and risk of complications in patients with type 2 diabetes (UKPDS 33). Lancet 1998;352:837-53.

7. Patel A, MacMahon S, Chalmers $\mathbf{J}$, et al. ADVANCE Collaborative Group. Intensive blood glucose control and vascular outcomes in patients with type 2 diabetes. N Engl J Med 2008;358:2560-72.

8. Duckworth W, Abraira C, Moritz T, et al. VADT Investigators. Glucose control and vascular complications in Veterans Affairs Diabetes Trial with type 2 diabetes. N Engl $J$ Med 2009;360: 129-39.

9. Gerstein HC, Miller ME, Byington RP, et al. ACCORD Investigators. Action to Control Cardiovascular Risk in Diabetes Study Group. Effects of intensive glucose lowering in type 2 diabetes. $\mathrm{N}$ Engl $\mathrm{J}$ Med 2008;358:2545-59.

10. Holman RR, Paul SK, Bethel MA, Matthews DR, Neil HA. 10-year follow-up of intensive glucose control in type 2 diabetes. N Engl J Med 2008;359:1577-89. [doi: 10.1056/NEJMoa0806470 ]

11. Hayward RA, Reaven PD, Wiitala WL, et al. Follow-up of glycemic control and cardiovascular outcomes in type 2 diabetes. N Engl J Med 2015;372:2197-206.

12. ACCORD Study Group. Nine-Year Effects of 3.7 Years of Intensive Glycemic Control on Cardiovascular Outcomes. Diabetes Care. 2016:39:701-8.

13. Wong MG, Perkovic V, Chalmers J, et al. ADVANCE-ON Collaborative Group. Long-term Benefits of Intensive Glucose Control for Preventing End-Stage Kidney Disease: ADVANCE-ON. Diabetes Care 2016;39:694700.

14. White WB, Cannon CP, Heller SR, et al. Alogliptin after acute coronary syndrome in patients with type 2 diabetes. The EXAMINE Trial. N Engl J Med 2013;369:1327-35.
15. Scirica BM, Bhatt DL, Braunwald E, et al. Saxagliptin and Cardiovascular Outcomes in Patients with Type 2 Diabetes Mellitus. N Engl J Med 2013;369:1317-26.

16. Green JB, Bethel MA, Armstrong PW, et al. Effect of Sitagliptin on Cardiovascular Outcomes in Type 2 Diabetes. N Engl J Med 2015;373:232-42.

17. Pfeffer MA, Claggett B, Diaz R, et al. Lixisenatide in Patients with Type 2 Diabetes and Acute Coronary Syndrome. N Engl J Med 2015;373:2247-57.

18. Zinman B, Wanner C, Lachin JM, et al. Empagliflozin, Cardiovascular Outcomes, and Mortality in Type 2 Diabetes. N Engl J Med 2015;373:2117-28.

19. Kernan WN, Viscoli CM, Furie KL, et al. Pioglitazone after Ischemic Stroke or Transient Ischemic Attack. N Engl J Med 2016;374:1321-31.

20. Marso SP, Daniels GH, Brown-Frandsen $\mathbf{K}$, et al. Liraglutide and Cardiovascular Outcomes in Type 2 Diabetes. N Engl J Med 2016;375:311-22.

21. Marso SP, Bain SC, Consoli A, et al. Semaglutide and Cardiovascular Outcomes in Patients with Type 2 Diabetes. N Engl J Med 2016;375: 1834-44.

22. Mann, J. Kidney Week 2016, Nov 15-20 2016, Chicago, IL.

23. Defronzo RA. Banting Lecture. From the triumvirate to the ominous octet: a new paradigm for the treatment of type 2 diabetes mellitus. Diabetes 2009;58:773-95.

24. Dormandy JA, Charbonnel B, Eckland DJ, et al. Secondary prevention of macrovascular events in patients with type 2 diabetes in the PROactive Study (PROspective pioglitAzone Clinical Trial In macroVascular Events): a randomised controlled trial. Lancet 2005;366:1279-89.

25. Nissen SE, Wolski $\mathbf{K}$. Effect of Rosiglitazone on the Risk of Myocardial Infarction and Death from Cardiovascular Causes. N Engl J Med 2007;356:2457-71.

26. Home PD, Pocock SJ, Beck-Nielsen H, et al. RECORD Study Team. Rosiglitazone evaluated for cardiovascular outcomes in oral agent combination therapy for type 2 diabetes (RECORD): a multicentre, randomised, open-label trial. Lancet 2009;373:2125-35.

27. Kernan WN, Inzucchi SE, Viscoli CM, et al. Impaired insulin sensitivity among nondiabetic patients with a recent TIA or ischemic stroke. Neurology 2003;60: 1447-51.

28. Wilcox R, Bousser MG, Betteridge DJ, et al. Effects of pioglitazone in patients with type 2 diabetes with or without previous stroke: results from PROactive (PROspective pioglitAzone Clinical Trial In macroVascular Events 04). Stroke 2007;38:865-73.

29. Erdmann E, Dormandy JA, Charbonnel B, Massi-Benedetti M, Moules IK. The effect of pioglitazone on recurrent myocardial infarction in 2,445 patients with type 2 diabetes and previous myocardial infarction: results from the PROactive (PROactive 05) Study. J Am Coll Cardiol 2007;49:1772-80.

30. Inzucchi SE, Viscoli CM, Young LH, et al. Pioglitazone Prevents Diabetes in Patients With Insulin Resistance and Cerebrovascular Disease. Diabetes Care 2016;39:1684-92.

31. DeFronzo RA, Tripathy D, Schwenke DC, et al. Pioglitazone for diabetes prevention in impaired glucose tolerance. N Engl J Med 2011;364:110415.

32. Gallo LA, Wright EM, Vallon V. Probing SGLT2 as a therapeutic target for diabetes: basic physiology and consequences. Diab Vasc Dis Res 2015; 12:78-89.

33. Fitchett D, Zinman B, Wanner C, et al. Heart failure outcomes with empagliflozin in patients with type 2 diabetes at high cardiovascular risk: results of the EMPA-REG OUTCOME® trial. Eur Heart J 2016;37:152634.

34. Wanner C, Inzucchi SE, Lachin JM, et al. Empagliflozin and Progression of Kidney Disease in Type 2 Diabetes. N Engl J Med 2016;375:32334.

35. American Diabetes Association. Standards of Medical Care in Diabetes 2017. Diabetes Care 2017;40:S1-S135. doi:10.2337/dc17-S001-S019.

36. Nauck MA, Meier JJ. The incretin effect in healthy individuals and those with type 2 diabetes: physiology, pathophysiology, and response to therapeutic interventions. Lancet Diabetes Endocrinol 2016;4:525-36.

37. Gerstein HC, Bosch J, Dagenais GR, et al. ORIGIN Trial Investigators. Basal insulin and cardiovascular and other outcomes in dysglycemia. N Engl J Med 2012;367:319-28.

38. Lipska KJ, Krumholz HM. Is hemoglobin Alc the right outcome for studies of diabetes? JAMA 2017:317:1017-18. 
39. Ismail-Beigi F, Moghissi E, Tiktin M, Hirsch IB, Inzucchi SE, Genuth S. Individualizing glycemic targets in type 2 diabetes mellitus: implications of recent clinical trials. Ann Intern Med 2011;154:554-9.
40. Inzucchi SE, Bergenstal RM, Buse JB, et al. Management of hyperglycemia in type 2 diabetes: a patient-centered approach: position statement of the American Diabetes Association (ADA) and the European Association for the Study of Diabetes (EASD). Diabetes Care 2012;35:1364-79. 